\title{
VALORAÇÃO MONETÁRIA: PESQUISAS EM FLORESTA URBANA
}

\author{
Sabrina Mieko Viana ${ }^{1}$, Larissa Leite Tosetti ${ }^{2}$, Luciana Cavalcante Pereira Rollo ${ }^{3}$, \\ Demóstenes Ferreira da Silva Filho ${ }^{4}$
}

(recebido em 19.09.2011 e aceito para publicação em 15.03.2012)

\section{RESUMO}

O uso de diferentes métodos para valoração monetária de bens e serviços ambientais é uma abordagem que pode trazer relevantes contribuições para planejamento de uso e a priorização de preservação das paisagens naturais. No caso das florestas urbanas, este tipo de pesquisa possuem potencial para sensibilização dos diversos setores da sociedade sobre os inúmeros serviços ambientais prestados por estas e seu papel essencial na qualidade ambiental urbana. Para avaliar como os diferentes métodos de valoração vêm sendo pesquisados no mundo, foram selecionados artigos publicados de 2001 a 2010, em 11 periódicos nacionais e internacionais para a confecção de um painel sobre as pesquisas nesta área. A maioria dos artigos publicados teve origem em estudos realizados na Europa e América do Norte, com predominância do uso da valoração hedônica, porém com tendência ao aumento de estudos sobre valoração contingente e dos métodos de fórmula. Concluiu-se que a diversidade de estudos sobre valoração deve ser estimulada, especialmente no contexto nacional, uma vez que não existe um método perfeito para valoração das florestas urbanas, em razão das múltiplas características e serviços prestados por estas. O entendimento do contexto, dos objetivos e limitações de cada método é fundamental para sua aplicação.

Palavras-chave: Métodos de valoração; Valoração econômica; Árvores urbanas; Serviços ambientais.

\footnotetext{
${ }^{1}$ Bióloga, doutoranda em Recursos Florestais pela Escola Superior de Agricultura "Luiz de Queiroz" sabrinamieko@gmail.com

${ }^{2}$ Eng. Agrônoma, mestranda em Recursos Florestais pela Escola Superior de Agricultura "Luiz de Queiroz" laletosetti@yahoo.com.br

${ }^{3}$ Eng. Agrônoma, doutoranda em Recursos Florestais pela Escola Superior de Agricultura "Luiz de Queiroz"-Irollo@usp.br

${ }^{4}$ Eng. Agrônomo, Prof. Dr. Departamento de Ciências Florestais - Escola Superior de Agricultura "Luiz de Queiroz" - dfilho@usp.br
} 


\section{MONETARY VALUATION: RESEARCHES IN URBAN FORESTRY}

\section{ABSTRACT}

The application of different methods in monetary valuation of natural goods and environmental resources is an approach that can bring relevant contributions for the planning as in the set of priorities for natural landscape preservation. In the case of the urban forests, this kind of research can be a useful tool to increase the society awareness about the numerous services provided by them and its essential role for the urban environmental quality. In order to assess how the different valuation methods have been used in in the world, we selected articles published in eleven international and Brazilian scientific journals, published from 2001 to 2011, aiming to make a panel about the studies about this theme. Most of the published papers were about researches conducted in Europe and North America, with predominant use of hedonic valuation method and a tendency, over time, to increase the use of contingent valuation and tree appraisal methods. We concluded that the diversity of monetary valuation researches should be encouraged, particularly in the Brazilian context, once there isn't only one perfect method for the urban forests assessment, given their multiple attribute and services provided by them. The understanding of the context, objectives and limitations of each valuation method is also essential for its application.

Keywords: Valuation methods, Economic valuation, Urban trees, Environmental services

\section{INTRODUÇÃO}

A valoração econômica de bens e serviços ambientais, com base no uso de ferramentas da economia, atualmente vem recebendo atenção por parte de pesquisadores e responsáveis das áreas de planejamento e gestão como forma de apoio na tomada de decisões, na avaliação de custos e benefícios, na alocação mais eficiente de recursos, na determinação de prioridades em projetos e políticas ou mesmo no cálculo de pagamentos por danos ambientais (MITCHELL; CARSON, 1989; LO; JIM, 2010). A partir desta valoração, obtemos uma medida monetária dos benefícios proporcionados pelos ativos 
ambientais e naturais, os quais são utilizados para a movimentação das atividades econômicas e humanas (MOTA, 2001).

Entretanto, essa atribuição de valores é uma tarefa de alta complexidade e até mesmo polêmica sob alguns pontos de vista. Uma das maiores dificuldades em se atribuir um valor econômico a estes se deve a algumas características, como a maioria destes não possuírem um mercado definido ou competitivo, serem afetados por externalidades ou serem bem públicos e portanto, não terem direito de propriedade bem definidos, gerando assim as falhas de mercado (KING et al., 2000; SCHAEFFER, 2008, THOMAS; CALLAN, 2010). Por estas razões, o mercado frequentemente não reflete todos os custos sociais e benefícios de um bem ou serviço ambiental, de forma que a alocação deste não será a mais benéfica ou eficiente (KING et al., 2000). Na tentativa de aproximar-se de uma adequada representação, várias pesquisas voltam-se para o desenvolvimento de diversos métodos de valoração monetária com diferentes abordagens, como determinar valores a partir da opinião de entrevistados, uso de mercados imobiliários, avaliações dendrométricas das árvores ou análises de custo-benefício, dentre outros.

Investigar o estado da arte destas pesquisas sobre valoração monetária das florestas urbanas é uma possibilidade de avaliar como estes métodos se desenvolvem, quais são mais utilizados e o comportamento do grau de interesse por este tipo de pesquisa ao longo do tempo, em diferentes países.

\section{O Urbano, Periurbano e as Florestas Urbanas}

A conceituação de termos é sempre um desafio quando se espera encontrar uma definição que melhor traduza a forma de entendimento do pesquisador para o assunto em discussão. Quando falamos em áreas urbanas e periurbanas, há uma grande gama de definições sobre sua dinâmica, delimitação e conceituação. Forman e Godron (1986) definem áreas urbanas como aquelas com grande adensamento de áreas construídas, que se estendem por quilômetros, ainda com a presença de pequenas manchas vegetadas, em forma de parques espalhados dentro delas, enquanto as áreas periurbanas seriam aquelas que misturam características rurais e urbanas, em uma mistura heterogênea de áreas residenciais, comerciais, plantações e áreas naturais. De forma geral, é possível enxergar as diferenças como gradientes, ou círculos concêntricos de influência que emanam de um centro urbano, onde a paisagem vai sendo modificada em consequência da interferência

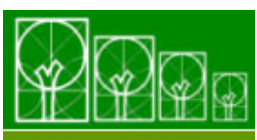

S $\cdot \mathbf{B} \cdot \mathbf{A} \cdot \mathbf{U}$ Soc. Bras. de Arborização Urbana 
humana (ANTROP, 2000). Estas alterações manifestam-se tanto na diferenciação do espaço, infraestrutura, uso, disponibilidade de serviços, como na mentalidade e percepção das pessoas que lá habitam.

Entretanto, não se pode deixar de denotar que estas definições ainda são muito discutidas, principalmente no que se diz respeito às áreas periurbanas, já que algumas de suas características, dinâmica, formação variam de um local para outro e também são dependentes de certos fenômenos, como a metropolização das cidades (NORONHA; HESPANHOL, 2008). No Brasil, podemos encontrar áreas periurbanas que englobam desde periferias com alta densidade populacional e condições precárias até aquelas onde se observam com maior clareza as transições do rural para o urbano.

Considerando que as florestas ultrapassam os limites políticos determinados pelos homens, localizando-se tanto em espaço urbano, quanto periurbano, rural e às vezes em mais de uma cidade, e que o espaço considerado como periurbano transforma-se rapidamente com o crescimento e expansão dos centros urbanos, optamos nesta revisão por adotar a definição dada por Moll (1995), onde a Floresta Urbana abrangeria as áreas onde já existem árvores ou com potencial para tal, dentro da área urbana e periurbana.

\section{Métodos de valoração monetária}

Segundo o ponto de vista defendido por Moll (1995), os formadores e gestores de políticas públicas ainda possuem pouca noção do valor monetário proporcionado pelas árvores quando consideradas como parte da infraestrutura urbana e não somente um mero "acessório estético". Pouco se leva em conta qual o valor econômico do papel destas na mitigação de enchentes, no controle de ilhas de calor, na melhoria da qualidade do ar, dentre outros fenômenos. Por esta razão, recentemente, o uso da valoração monetária nas florestas urbanas tem ganhado força, sendo os métodos mais utilizados nesta área estão a valoração hedônica, a valoração contingente e os métodos de valoração por fórmulas (tree appraisal methods).

$\mathrm{Na}$ valoração hedônica, leva-se em conta o efeito de um dado benefício ambiental sobre o preço de um bem que possua um mercado real. Especificamente no caso das florestas urbanas, vários estudos têm trabalhado com a relação do valor de mercado imobiliário, ou seja, o quanto o valor de um imóvel pode ser influenciado pela simples proximidade de uma área bem arborizada, por meio de fórmulas que levarão em conta

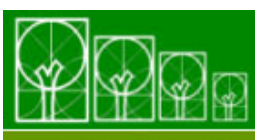

$\mathbf{S} \cdot \mathbf{B} \cdot \mathbf{A} \cdot \mathbf{U}$ Soc. Bras. de Arborização Urbana 
determinadas características dos bens mercantis e informações de seu mercado (SANDER et al., 2010). A base deste método é a valorização dada pelos consumidores a certas características e serviços ambientais, mais do que o valor do bem (que possui mercado) em si mesmo (KING et al., 2000). Sua principal vantagem é a possibilidade de comparação com preços de mercado; porém trata-se de um método cujos resultados são dependentes da fórmula adotada e de interpretação relativamente complexa.

O método da valoração contingente (MVC) é um método direto, baseado no valor de uso passivo, cuja utilização vem aumentando nas últimas décadas especialmente na valoração de recursos naturais e bens públicos (CARSON, 2000; MOTA, 2001). Estes bens podem ser valorados por meio da "disposição a pagar" ou pela "disposição em receber" manifestada. No caso da disposição a pagar, a intenção é descobrir qual o valor máximo que um indivíduo está "disposto a pagar" para ter acesso ou usufruir de um bem ou serviço ambiental enquanto na "disposição em receber", procura-se saber qual o valor mínimo que um indivíduo está disposto a receber para abrir mão de um bem (LO; JIM, 2010).

Dentre as principais vantagens deste método está a flexibilidade, pois pode ser usado para valorar tanto valores de uso como de não-uso. Geralmente é mais utilizado no segundo caso, pois nesse tipo de método pode-se criar um mercado hipotético para o bem ou serviço ambiental a ser avaliado (KING et al., 2000). Entretanto, este método recebe severas críticas devido ao uso da opinião direta dos entrevistados e a incerteza da real compreensão destes sobre os valores inquiridos.

Watson (2002) apresentou os métodos de fórmula (tree appraisal methods) divididos em dois tipos: fórmulas que estabelecem um valor inicial baseado no tamanho das árvores e ajusta por fatores de condição, local, espécie, qualidade e situações especiais, ou o valor determinado a partir de classificação de pontos para fatores (multiplicados ou somados) com fator monetário introduzido no final (geralmente ligadas a fatores de mercado como preço da muda e preço de plantio ou consenso).

Dentro do acima exposto procurou-se, neste artigo, quantificar e levantar a distribuição dos métodos de valoração da floresta urbana que têm sido utilizados em pesquisas publicadas nos últimos 10 anos (2001-2010), em quais países elas tem sido realizadas, a partir de uma seleção de 11 periódicos de abrangência nacional e internacional. 


\section{MATERIAIS E MÉTODOS}

Foram levantados os artigos sobre valoração monetária da floresta urbana e periurbana das edições dos anos de janeiro 2001 a dezembro 2010 das seguintes revistas internacionais e nacionais: Journal of Environmental Management, Ecological Economics, Urban Forest and Urban Greening, Landscape and Urban Planning, Forestry, Revista Brasileira de Arborização Urbana (REVSBAU), Árvore, Scientia Forestalis, Ciência Florestal, Scientia Agricola e Cerne.

A seleção das cinco revistas internacionais foi baseada na relevância de suas publicações para as pesquisas voltadas ao meio ambiente urbano e sua floresta. Também foi feito um levantamento em cinco revistas nacionais, consideradas como de maior impacto no estudo das Ciências Florestais (Árvore, Scientia Forestalis, Ciência Florestal, Scientia Agricola e Cerne) e a REVSBAU como uma das únicas publicações voltada somente para o estudo das Florestas Urbanas.

As publicações encontradas foram separadas em quatro categorias, conforme critérios descritos a seguir:

\section{Método de valoração}

- Valoração hedônica: pesquisas de valoração da floresta urbana que referiram-se sobre a relação desta com custo de bens mercantis, como o custo de venda de imóveis;

- Valoração contingente: nesta categoria foram inclusos estudos que utilizaram métodos de valoração e escolha contingente;

- Custo de viagem: quando se levaram em consideração custos de deslocamento até dado parque, espaço verde, dentre outros;

- Custo-benefício: pesquisas que analisaram os benefícios da floresta urbana em relação aos custos para gerenciamento e manutenção;

- Métodos de fórmula: estudos com utilização de métodos baseados em fórmulas para estimativa do valor individual de árvores com base em seu custo de implantação e manutenção ou em relação as suas características;

- Revisão: artigos de revisão sobre métodos variados de valoração monetária;

- Outros: foram incluídos nesta categoria tipos de valoração que não se encaixaram nas demais categorias, como análise de multicritério e análise de custos de implantação em relação a aplicação de multas. 


\section{Região da floresta valorada}

- Floresta urbana;

- Floresta periurbana, que está englobada no conceito de Floresta Urbana supracitado, mas quantificadas com base nos artigos analisados.

\section{Ano de publicação do artigo}

Distinguiu-se esta categoria para avaliação da utilização dos métodos de valoração ao longo do tempo.

\section{Local das pesquisas}

Dentre os artigos selecionados também foram levantados os países nos quais os estudos de valoração foram elaborados.

\section{RESULTADOS E DISCUSSÃO}

Ao todo foram levantados 37 artigos sobre valoração de florestas urbanas, seis deles em revistas brasileiras e 31 em revistas internacionais. A maioria das pesquisas utilizou métodos de valoração hedônica, métodos de valoração contingente, seguidos do uso de métodos de fórmula e da análise de custo-benefício (Figura 1). 
Figura 1. Porcentagem de artigos publicados em relação ao método de valoração empregado na pesquisa. Legenda: VH=valoração hedônica, VC=valoração contingente, $\mathrm{MF}=$ método de fórmula, $\mathrm{CB}=$ custo benefício, $\mathrm{Re}=$ revisão, $\mathrm{CV}=$ custo de viagem

Figure 1. Percentage of articles published, in relation to valuation method employed in the research. Legend: $\mathrm{VH}=$ hedonic valuation, $\mathrm{VC}=$ contingent valuation, $\mathrm{MF}=$ tree appraisal formulas, $\mathrm{CB}=$ cost-benefit, $\mathrm{Re}=$ revision, $\mathrm{CV}=$ travel cost

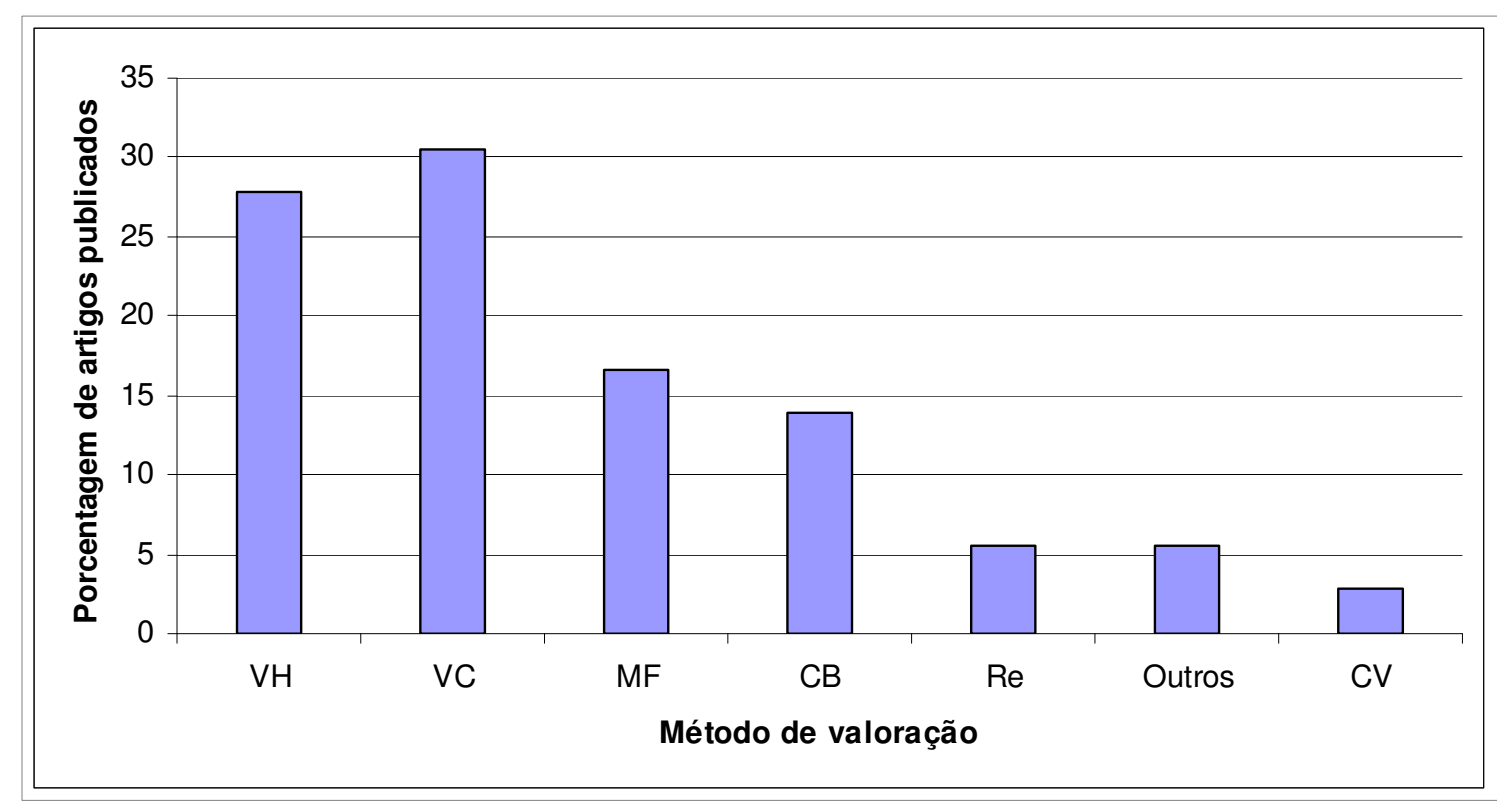

A análise da freqüência de artigos ao longo do tempo revela o aumento das pesquisas sobre valoração no período de 2006 a 2010, com a predominância do uso dos métodos de valoração hedônica e contingente. Entretanto, nota-se neste mesmo intervalo de tempo, um aumento considerável de estudos com a utilização de métodos de fórmula (Figura 2). 
Figura 2. Evolução temporal da frequência de artigos publicados em relação ao método de valoração empregado na pesquisa. Legenda: VH=valoração hedônica, $\mathrm{VC}=$ =valoração contingente, $\mathrm{MF}=$ =método de fórmula, $\mathrm{CB}=$ custo benefício, $\mathrm{Re}=$ revisão, $\mathrm{CV}=$ custo de viagem

Figure 2. Temporal evolution of the frequency of papers published, related to valuation method applied in the research. Legend: $\mathrm{VH}=$ hedonic valuation, $\mathrm{VC}=$ contingent valuation, $\mathrm{MF}=$ tree appraisal method formula, $\mathrm{CB}=\cos$ benefit, $\mathrm{Re}=$ revision, $\mathrm{CV}=$ travel cost

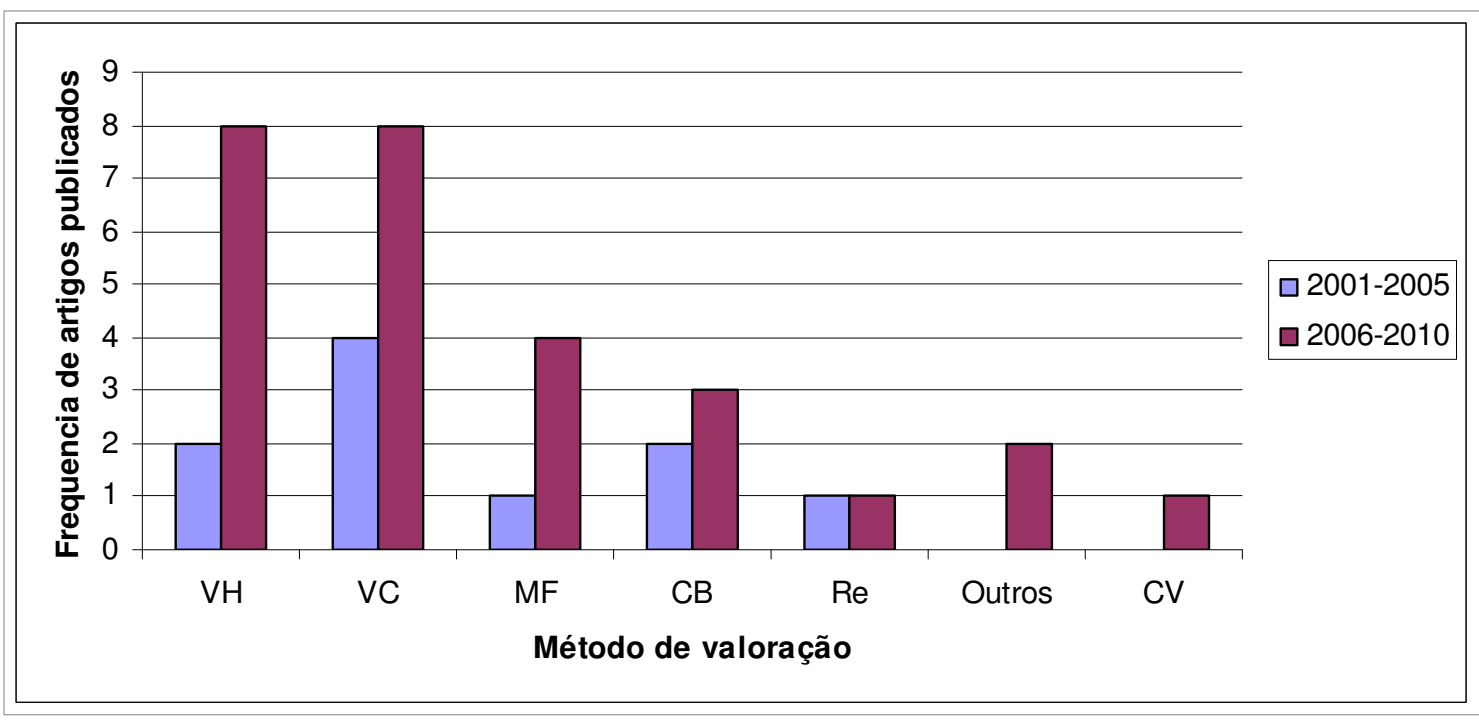

McPherson e Simpson (2002) indicaram que tanto a valoração contingente como a valoração hedônica captam o valor do conjunto, porém são limitadas quando se quer obter o valor individual, tanto em termos de custo quando de benefício, de cada árvore.

Artigos cujo estudo de valoração focaram-se em florestas localizadas nas áreas urbanas são maioria em comparação com aqueles realizados em florestas periurbanas ou ainda que englobaram os dois tipos de florestas (Tabela 1). 
Tabela 1. Freqüência de utilização dos diferentes métodos de valoração monetária em relação ao tipo de floresta estudada

Table 1. Frequency of use of different methods of monetary valuation, in relation to the studied forest type

\begin{tabular}{l|c|c|c|c}
\cline { 2 - 4 } \multicolumn{1}{c|}{ Método de valoração } & Urbana & Periurbana & $\begin{array}{r}\text { Urbana e } \\
\text { Periurbana }\end{array}$ & TOTAL \\
\hline Custo de viagem & 0 & 0 & 1 & 1 \\
\hline Custo-benefício & 4 & 0 & 1 & 5 \\
\hline Método de fórmula & 4 & 1 & 0 & 5 \\
\hline Valoração contingente & 10 & 1 & 1 & 10 \\
\hline Valoração hedônica & 8 & 1 & 1 & 10 \\
\hline Revisão & 0 & 1 & 1 & 2 \\
\hline Outros & 1 & 1 & 0 & 2 \\
\hline \multicolumn{1}{c|}{ TOTAL } & $\mathbf{2 7}$ & $\mathbf{5}$ & $\mathbf{5}$ & $\mathbf{3 7}$ \\
\hline
\end{tabular}

Com relação aos locais de estudo, a maior parte das pesquisas selecionadas foram realizadas no Brasil $(5 ; 13,51 \%)$, Estados Unidos $(11 ; 29,73 \%)$ e China $(3 ; 8,11 \%)$. A quantidade significativa de estudos no Brasil deve-se à contribuição das revistas nacionais. $\mathrm{Na}$ categoria "Outros" foram contabilizados países cuja frequência de artigos foi igual ou menor a 2, a saber: Reino Unido, Suíça, Dinamarca, Japão, Chile, Hong Kong, Espanha, Holanda, Austrália, Finlândia, Nova Zelândia e Itália.

A valoração monetária de florestas urbanas pode ser um meio de sensibilizar o setor público, privado e os moradores das cidades da ordem de grandeza representada pela presença de florestas nos centros urbanos, apontando os bens e serviços proporcionados. Por exemplo, McPherson e Simpson (2002), relataram que seu estudo sobre o custobenefício das florestas urbanas das cidades norte-americanas de Santa Monica e Modesto, além de sensibilizar a população sobre a importância da manutenção das árvores das vias públicas e parques, também funcionou como argumento para a manutenção dos fundos para o cuidado com elas, em contraste com outras cidades onde houve corte destas verbas.

Entretanto, quando se trata de um tema tão polêmico quanto a atribuição de valores a bens e serviços naturais, deve-se considerar que atores subjetivos, simbolismos e sentimentos associados a determinados bens naturais também podem colocá-los como 
impossíveis de serem valorados em termos econômicos, como no exemplo dado Schaeffer (2008), sobre os territórios considerados como sagrados por indígenas norte-americanos. Além disso, em alguns casos, a perda de certa eficiência econômica em relação ao gerenciamento de bens e serviços ambientais é altamente justificável, como por exemplo, quando há a participação política das comunidades envolvidas no controle e usufruto destes.

A compreensão dessas técnicas e das diferentes formas de valoração, o entendimento das suas propriedades, as bases filosóficas e teóricas são importantes, já que são elas que fundamentam quais as limitações de cada método (SCHAEFFER, 2008). Portanto, não existe um único método que seja perfeito para valoração de todas as múltiplas características e serviços prestados pelas florestas urbanas; a aplicação dos mesmos dependerá dos objetivos e do contexto local.

\section{CONCLUSÕES}

Dentre os artigos analisados, houve a predominância da utilização dos métodos de valoração contingente e hedônica, porém com certa tendência ao aumento de pesquisas com uso de métodos de fórmula e uma quantidade significativa de estudos realizada com foco nas florestas urbanas. Grande parte das contribuições no campo da valoração das florestas urbanas ainda está concentrada em países da América do Norte e da Europa, e os poucos estudos realizados no Brasil foram publicados somente em revistas de escopo nacional.

Concluímos assim que a diversidade de estudos sobre valoração deve ser estimulada, uma vez que não existe um método perfeito para valoração das florestas urbanas, dadas as múltiplas características e serviços prestados por estas. O entendimento do contexto, dos objetivos e limitações de cada método é fundamental para sua aplicação.

Valorar a floresta urbana em termos econômicos pode ser uma ferramenta para sensibilização do setor público, privado e os moradores das cidades sobre a ordem de grandeza representada pela presença de florestas nos centros urbanos, apontando os bens e serviços proporcionados e garantindo seu espaço junto com as demais estruturas urbanas.

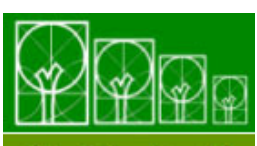




\section{REFERÊNCIAS}

ANTROP, M. Background concepts for integrated landscape analysis. Agriculture Ecosystems and Environment, v.77, n.1-2, p.17-28, 2000.

CARSON, R.T. Contingent Valuation user's guide. Environmental Science and Technology, n. 34, p. 1413-1418, 2000.

FORMAN, R.T.T.; GODRON, M. Landscape ecology. New York: Willey. 1996, 640p.

KING, D. M.; MAZZOTTA, M. J.; MARKOWITZ, K.J. Ecosystem Valuation, USDA / NRCS / NOAA, 2000. Disponível em: <http://ecosystemvaluation.org>. Acesso em: 1 set. 2010.

LO, A. Y.; JIM, C.Y. Willingness of residents to pay and motives for conservation of urban green spaces in the compact city of Hong Kong. Urban Forestry and Urban Greening, v.9, n.2, p.113-120, 2010.

McPHERSON, E. G.; SIMPSON, G. R. A comparison of municipal forest benefits and costs in Modesto and Santa Monica. Urban Forestry and Urban Greening, v.1, p.61-74, 2002.

MOLL, G. Urban forestry: a national initiative. In: BRADLEY, G.A., (ed.) Urban forest landscapes: integrating multi-disciplinary perspectives. University of Washington Press: Seattle, WA., 1995, pp. 12-16.

MOTTA, J.A. O valor da natureza: economia e política dos recursos naturais. Rio de Janeiro, RJ: Garamond. 2001.198p.

MITCHELL, R.C.; CARSON, R.T. Using surveys to value public goods: The contingent valuation method. RFF Press, 1989, $470 \mathrm{p}$.

NORONHA, E.O.; HESPANHOL, R.A.M. O espaço periurbano no município de Jundiaí-SP: Características e tendências atuais. Revista Formação, v.1, n.15, p.85-96, 2008. 
SANDER, H.; POLASKY, S.; HAIGHT, R.G. The value of urban tree cover: a hedonic property model in Ramsey and Dakota Counties, Minnesota, USA. Ecological Economics v. 69, n.8, p.1646-1656, 2010.

SCHAEFFER, P.V. Thoughts concerning the economic valuation of landscapes. Journal of Environmental Management, v.89, n.3 , p. 146-154. 2008.

THOMAS, J.M.; CALLAN, S.J. Economia Ambiental: aplicações, políticas e teoria. São Paulo: Cengage Learning, 2010. $556 \mathrm{p}$.

WATSON, G. Comparing formula methods of tree appraisal. Journal of Arboriculture, v.28, n.1, p.11-18, 2002. 